\title{
Lectura en papel y digital en la biblioteca infantil: una convivencia necesaria
}

\author{
Raquel Gómez Díaz \\ Universidad de Salamanca, España \\ rgomez@usal.es
}

Araceli García-Rodríguez

Universidad de Salamanca, España

araceli@usal.es

Cita sugeridaGómez Díaz, R. y García-Rodríguez, A. (2018). Lectura en papel y digital en la biblioteca infantil: una convivencia necesaria.Palabra Clave (La Plata), 7(2), e045. https://doi.org/10.24215/18539912e045 


\title{
Lectura en papel y digital en la biblioteca infantil: una convivencia necesaria
}

\author{
Paper and digital reading in children's libraries: a necessarry coexitence \\ Raquel Gómez Díaz \\ Universidad de Salamanca, España \\ rgomez@usal.es \\ Araceli García-Rodriguez \\ Universidad de Salamanca, España \\ araceli@usal.es
}

\section{Resumen:}

Uno de los retos de los bibliotecarios infantiles del siglo XXI es integrar la colección y las actividades de la biblioteca con los nuevos contenidos y actividades procedentes del entorno digital. De esta manera se puede favorecer el aprendizaje y disfrute en la biblioteca, aprovechando la oportunidad de mejorar los productos y servicios e incluso atraer a nuevos usuarios. Para realizar esto con éxito es necesario conocer, seleccionar, adquirir, integrar, visibilizar y dinamizar lo analógico y lo digital, identificando las actividades que cumplirán cada una de estas funciones, que es precisamente lo que se hace en este artículo.

Palabras clave: Lectura digital, Aplicaciones de lectura, Bibliotecas infantiles, Dinamización de contenidos digitales, Promoción de la lectura.

\section{Abstract:}

One of the challenges that the child librarians of the 21 st century is to integrate the collection and the activities of the library with the new contents and events from the digital environment. In this way, you can promote learning and enjoyment in the library, taking advantage of the opportunity to improve products and services and even attract new users. To archived this successfully, it is necessary to know, select, acquire, integrate, visualize and dynamize the analog and the digital, identifying the activities that will fulfill each of these functions, which is what it does in this article.

KEYWORDS: Reading app, Children library, Digital content dynamization, Digital literacy and library.

\section{INTRODUCCIÓN}

Desde hace unos cuantos años vemos cómo la variedad de elementos que integran la biblioteca crece sin parar, como la música y los vídeos. Los programas de ordenador se han ido integrando en la biblioteca con el único fin de dar respuesta a las necesidades y preferencias cambiantes de los usuarios que, en muchos casos, pasan por lo digital (Gómez-Díaz \& García-Rodríguez, 2015). No responder a estas nuevas necesidades puede "ser un suicidio para instituciones que necesitan la percepción y valoración por parte de la sociedad para reafirmar su papel en la misma" (Gallo León, 2013, p.52).

La incorporación de la tecnología a la biblioteca no debe verse como un problema sino como una oportunidad de mejorar los productos y servicios, y por qué no, también de atraer a nuevos lectores, ampliando con "nuevos servicios, nuevos objetivos, nuevos medios de comunicación y difusión para llegar a las personas, de sumar todo aquello que haga a la biblioteca ser una entidad referente dentro de la comunidad" (Marquina, 2013, p. 10).

Sabemos que a los más pequeños les apasiona la tecnología y esta puede ser un medio para atraer nuevos lectores (García-Rodríguez y Gómez-Díaz, 2017a). Lo digital no tiene por qué competir con el papel, son simplemente respuestas distintas a la manera de leer.

Cuando surgió la lectura digital muchos la rechazaron, y otros la siguen rechazando, quizá en algunos casos por el desconocimiento de toda su potencialidad para la promoción lectora. Y es que, para tener una opinión formada de algo, en este caso la lectura digital, es necesario conocer sus posibilidades; es aquí donde 
la biblioteca puede y debe convertirse en un agente promotor, haciendo que todos los usuarios conozcan los nuevos soportes y tipos de contenidos, a través de la integración de estos, con los contenidos físicos presentes en las bibliotecas desde hacer años.

Para que la incorporación de lo digital se produzca en las mejores condiciones, es necesario hacer cambios en el modelo tradicional de biblioteca, ampliando la oferta de tipos de formatos y productos para que esta se convierta en centro de información, conocimiento, recursos y actividades, que cuide al máximo la interacción con los usuarios y su participación activa y que aproveche las oportunidades de espacios webs, contenidos y canales digitales ("Aprovechar las TIC en la biblioteca infantil," 2016).

Es evidente que la lectura digital requiere de nuevas formas de trabajar, nuevas competencias propias de un sistema intermediado tecnológicamente en el que los dispositivos de lectura y las formas de esta exigen comportamientos y actitudes de los usuarios que es preciso conocer y trabajar (Alonso-Arévalo, GómezDíaz y Cordón-García, 2015), lo que obliga a las bibliotecas a adaptar lo que tradicionalmente se venía haciendo. A las diferentes actividades que se han venido realizando durante años, hay que sumar aquellas realizadas de manera no presencial y otras en las que se trabaje con diferentes dispositivos, consiguiendo así que los usuarios conozcan todas sus potencialidades y se apropien de ellos, trabajando así para que los lectores tradicionales sean también digitales (Cordón-García, 2016), al tiempo que se abren las puertas de la biblioteca para conseguir que los usuarios que no pueden acudir al centro puedan participar también del día a día de la biblioteca.

Pero no se trata simplemente de sumar, sino también de integrar, conseguir que la biblioteca sea un espacio híbrido donde la experiencia del pasado se alimente con la novedad de lo tecnológico, y donde esto último se enriquezca y se asiente con las prácticas anteriores. Digital y papel, presencial y online, bibliotecario y usuario no son binomios contrapuestos sino piezas del engranaje de la biblioteca del futuro que deben estar bien engrasados con la formación, la incorporación de contenidos y soportes y su dinamización, elementos que permitirán conseguir esta deseada integración y ofrecer servicios novedosos y adaptados a la sociedad digital.

Las bibliotecas infantiles han sido siempre un ejemplo de centros activos, en los que los espacios y los servicios se han renovado continuamente para adaptarse a las necesidades e intereses de un grupo de usuarios, en el que no solo están los niños, sino también padres y profesores, una adaptación que en los últimos años se está dirigiendo precisamente a este laberinto digital (Gómez-Díaz, García-Rodríguez, Cordón-García y Alonso-Arevalo, 2016).

Ante la variedad de experiencias que en los últimos años se vienen realizando en la biblioteca infantil, es necesario proceder a una sistematización de acciones que sirva de guía a los profesionales que pretenden avanzar en la dinamización on line de los contenidos, sean digitales o no, y por otro en la dinamización presencial de los contenidos digitales. En este último caso, una estrategia que servirá además para potenciar el uso del espacio físico de la biblioteca.

\section{OBJeTivo y METOdología}

El objetivo del presente artículo consiste en determinar las fases que debe adoptar una biblioteca infantil para conseguir una integración completa de lo físico y lo digital, y además, proponer actividades y servicios permitan dicha integración.

El primer paso para la realización de este trabajo fue la búsqueda de experiencias desarrolladas en bibliotecas. Muchas de ellas se cuentan en post de blog de bibliotecas, o aparecen como noticias en blogs especializados en el ámbito digital o en literatura infantil, pero la falta de normalización en el etiquetado de estos posts, o incluso su ausencia, dificultó el proceso de localización. Las búsquedas en bases de datos generales y especializadas, no aportó resultados. 
Una vez localizadas las diferentes estrategias, se procedió a su clasificación en torno a una secuencia lógica de acciones que se consideran esenciales para planificar un programa de actividades de integración de lo físico y lo digital, de lo presencial y lo virtual: conocer, seleccionar, adquirir, integrar, visibilizar y dinamizar.

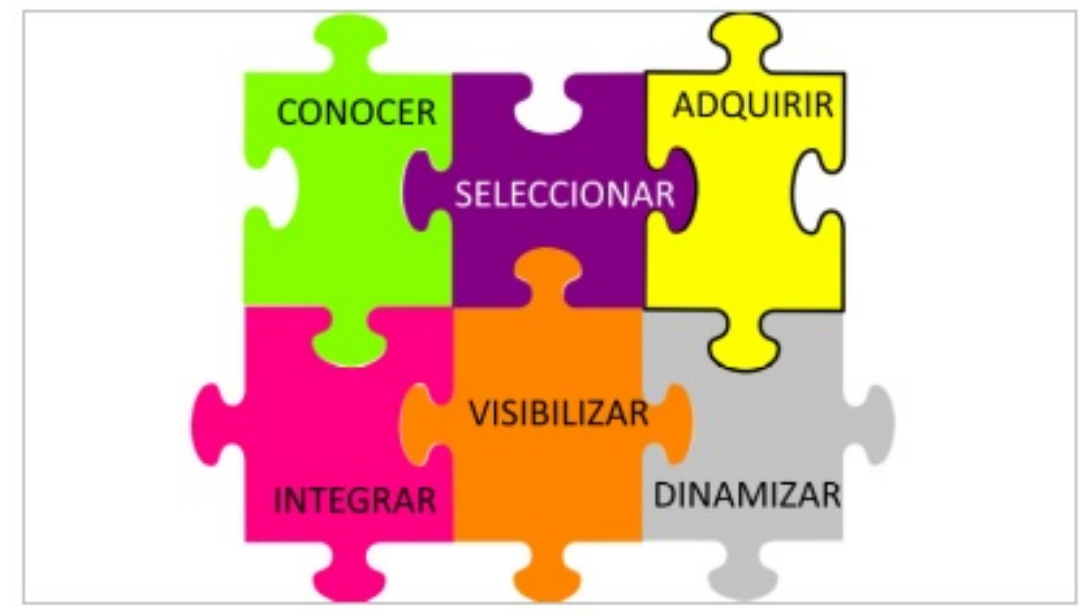

FIGURA 1

Acciones encaminadas a la construcción de la biblioteca del futuro

Fuente: elaboración propia

A cada una de estas acciones corresponden distintas actividades, muchas de las cuales pueden corresponderse a más de una acción. Para una mayor claridad, se procedió a representarlas en una tabla que sirvió de base para la descripción detallada de cada una de ellas.

\begin{tabular}{|c|c|c|c|c|c|c|}
\hline & Conocer & Seleccionar & Adquirir & Integrar & Visibilizar & Dinamizar \\
\hline $\begin{array}{l}\text { Diseño de un plan } \\
\text { integrado de } \\
\text { colecciones }\end{array}$ & & $\mathrm{x}$ & $\mathrm{x}$ & $\mathrm{x}$ & & \\
\hline $\begin{array}{l}\text { Selección de las } \\
\text { fuentes de } \\
\text { información }\end{array}$ & $\mathrm{x}$ & $\mathrm{x}$ & & & & \\
\hline $\begin{array}{l}\text { Integración de } \\
\text { contenidos } \\
\text { digitales en el } \\
\text { catálogo }\end{array}$ & & & & $\mathrm{x}$ & $\mathrm{x}$ & \\
\hline $\begin{array}{l}\text { Señalizar los } \\
\text { contenidos }\end{array}$ & & & & $\mathrm{x}$ & $\mathrm{x}$ & \\
\hline $\begin{array}{l}\text { Actividades de } \\
\text { formación }\end{array}$ & $\mathrm{x}$ & $\mathrm{x}$ & & & $\mathrm{x}$ & $\mathrm{x}$ \\
\hline $\begin{array}{l}\text { Recomendación de } \\
\text { contenidos }\end{array}$ & $x$ & $x$ & & $x$ & $x$ & $x$ \\
\hline Hora del cuento & & & & & $x$ & $x$ \\
\hline Club de lectura & & & & & $\mathrm{x}$ & $\mathrm{x}$ \\
\hline Gamificación & & & & & $x$ & $\mathrm{x}$ \\
\hline Préstamo & $\mathrm{x}$ & & & & $\mathrm{x}$ & $x$ \\
\hline
\end{tabular}

TABLA 1

Acciones y actividades de integración de contenidos

elaboración propia 


\section{Desarrollo de acciones y estrategias en la biblioteca}

En este apartado se describirán de forma más detallada cada una de las acciones con sus correspondientes actividades, siempre teniendo en cuenta que esto es solo una muestra que sin duda se irá enriqueciendo con las aportaciones de los diferentes bibliotecarios.

\subsection{CONOCER, SELECCIONAR Y ADQUIRIR}

En este primer epígrafe se agrupan las actividades en torno a la formación de la colección.

Una de las características del mundo infantil es la gran variedad de contenidos, a los que ahora hay que sumar un gran abanico de productos digitales. Unos son el resultado de un mero cambio de soporte, otros incluyen un amplio catálogo de enriquecimientos (enlaces hipertextuales, animación de objetos y personajes, sonido...) que se plasman en distintos productos (videolibros, apptoy, libros app), como se puede comprobar en García-Rodríguez y Gómez-Díaz (2016a).

Por ello es inviable el desarrollo de actividades relacionadas con los contenidos digitales sin que el bibliotecario tenga unos conocimientos básicos, no solo de los productos con los que está trabajando, como ser tipos de contenidos, fuentes y recursos para la adquisición y selección, modelos de negocio y funcionalidades entre otras cosas, sino también de las últimas innovaciones tecnológicas y su viabilidad de incorporación a la biblioteca.

El primer paso es elaborar un plan de desarrollo de colecciones en el que definamos no solo la estructura y formato de la colección, los criterios cuantitativos y cualitativos de selección, modelos y métodos de adquisición (compras individuales y en bundles, integradas, ofertas, etc. Véase García-Rodríguez y GómezDíaz, 2016b), las prioridades, etc.

En lo que se refiere a la elección de las fuentes de información, son varios los recursos que se pueden utilizar. Para los contenidos digitales: blogs, plataformas de venta y distribución, desarrolladores, tiendas de aplicaciones, recomendadores, premios especializados, etc. (García-Rodríguez y Gómez-Díaz, 2016b). En el caso de los libros impresos, se puede además consultar revistas, web de las editoriales, centros de documentación especializados, web de instituciones y asociaciones, entre otros (García-Rodríguez y GómezDíaz, 2017b).

\subsection{INTEGRAR}

La primera fase de formación culmina con una doble integración de los contenidos digitales; por un lado, en el instrumento de búsqueda por excelencia de una biblioteca, el catálogo, y por otro, con la colección física en las estanterías.

Es necesario que los contenidos digitales se integren en el catálogo. Diversos estudios han demostrado que la incorporación de los registros bibliográficos de los e-books en el catálogo puede incrementar su uso hasta en un 50\% (E-book collection management, 2009). De esta forma, el usuario puede acceder con una sola búsqueda a toda la colección independientemente del soporte y formato; una acción que facilitará la localización de la obra, al no tener que buscar en dos plataformas diferentes y dirigirse directamente desde el registro a la plataforma de préstamo. En el caso de disponer de libros app es recomendable incorporar la descripción al propio catálogo, independientemente de que en los datos de localización se indique el dispositivo en el que aparece precargada.

La integración se puede hacer visible convirtiendo el catálogo en un escaparate, como propone la nueva versión de AbsysNet 2.2, que permite visualizar todos los contenidos del OPAC mediante la incorporación de un carrusel de cubiertas en la página inicial del catálogo ("El carrusel de portadas", 2017). 
Por otro lado, existe la posibilidad de integrar las colecciones en el espacio físico de la biblioteca a través de una correcta señalización; una estrategia especialmente útil para los niños que habitualmente buscan en la propia estantería. Se trata en este caso de proporcionar una representación visual de los títulos disponibles en formato electrónicos.

Existen varias opciones:

a) Cuando no se disponga de ejemplar en formato físico, intercalar tarjetas de estante con la información del contenido digital, en el lugar de la estantería que ocuparía el ejemplar en papel. En la tarjeta se incorpora la imagen de cubierta y un código QR que le dirige al catálogo para que pueda descargarlo directamente.
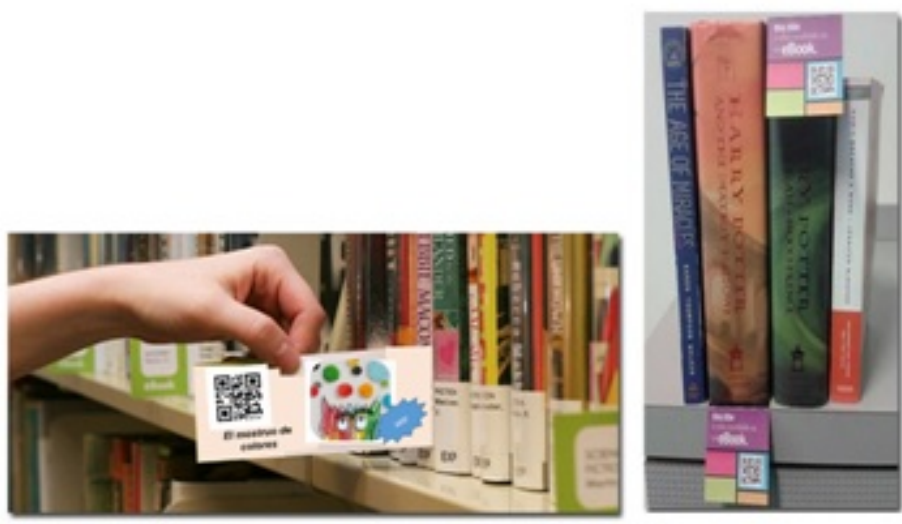

FIGURA 2

Tarjetas de estante con código QR

Fuente: elaboración propia

a) Cuando disponemos de ambos formatos, se puede incluir una pegatina en el ejemplar impreso o una tarjeta en el estante o en el propio libro, indicando su disponibilidad, ebook, app, etc., siempre incluyendo el código QR

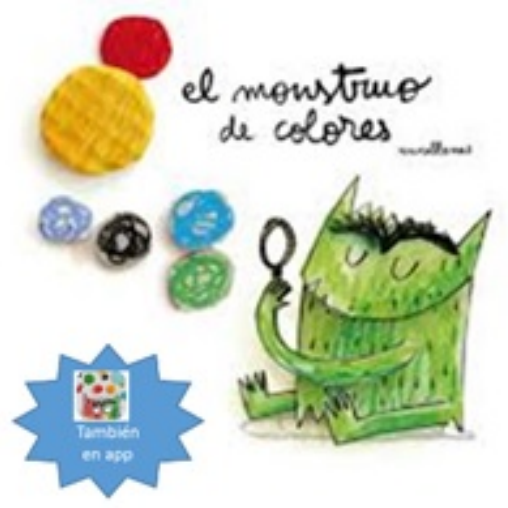

FIGURA 3

Disponibilidad de un título en formato app

Fuente: elaboración propia, basado en El monstruo de colores

a) Colocar códigos QR en los muros de la biblioteca para que cualquier usuario con carnet pueda desde el exterior acceder al catálogo y hacer el préstamo. 
A través de estas acciones se fomenta la integración de las colecciones, pero también se hace más visible y facilita el acceso conjunto a los usuarios.

\subsection{Y TRAS INTEGRACIÓN VISIBILIZAR LAS COLECCIONES}

Aunque en sí misma la integración de los contenidos es ya una forma de visibilizarlos, la biblioteca puede llevar a cabo acciones específicas con este objetivo, y para ello debe empezar con algo tan básico como la difusión a través de blogs, redes sociales y boletines del centro, e incorporar un servicio de aviso o alerta informativa a través de la aplicación móvil de la biblioteca, canales RSS u otros medios.

Un recurso para facilitar la visibilidad y el descubrimiento de los títulos puede ser la instalación de pantallas táctiles, como por ejemplo OverDrive Media Station, desde las que se puede visualizar cualquier título y enviarlo al dispositivo de lectura. Esta opción permite seguir manteniendo usuarios presenciales, es decir, se efectúa un préstamo digital pero dentro del espacio físico de la biblioteca.

Sin embargo, los contenidos infantiles deben ser presentados de una forma diferente, por lo que es recomendable que se utilicen herramientas de descubrimiento específicas, personalizadas y simplificadas en las que se puedan incluir títulos adecuados a cada etapa lectora y a las características de este tipo de usuarios, como se realiza en la Kitsap Regional Library de eReading Room.

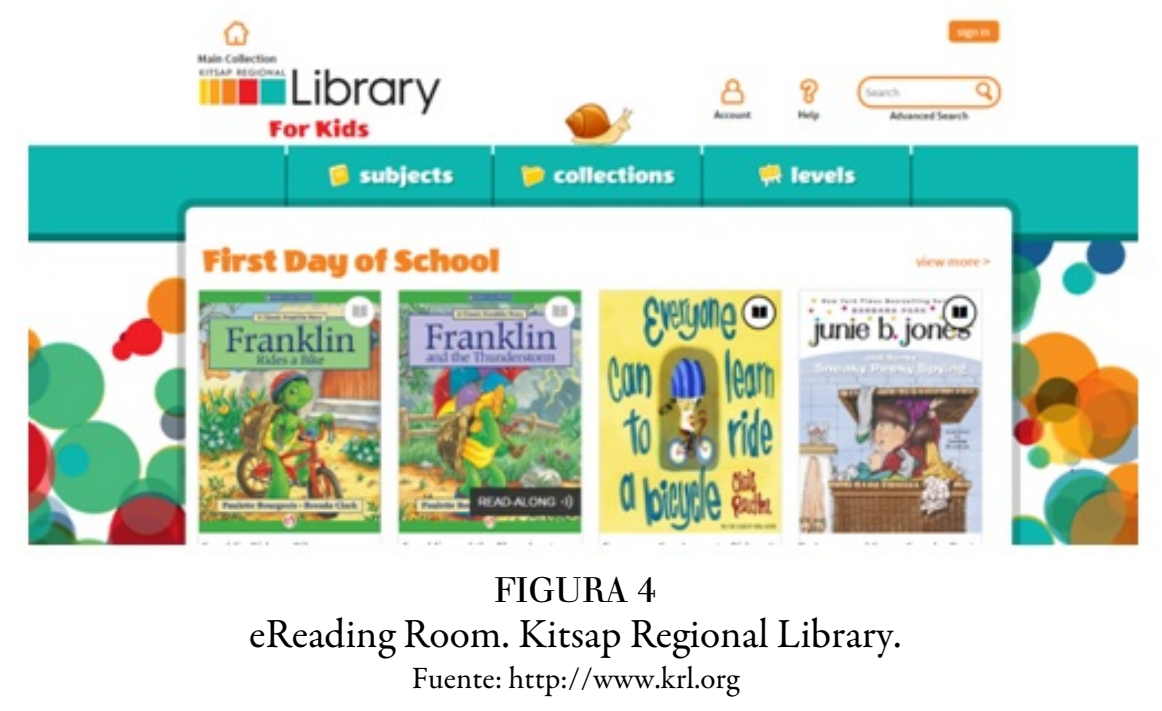

Si la biblioteca utiliza marcapáginas recordatorio de la fecha de devolución del ejemplar físico, se puede incluir en este un código QR que apunte a la sección de novedades de la web de la biblioteca, con lo que los usuarios siempre tendrán acceso a esa página de manera directa. Simplemente es necesario hacer una renovación regular de los contenidos de novedades, pero no será necesario crear nuevos códigos $\mathrm{QR}$.

\subsection{Y FINALMENTE DINAMIZAR}

El fomento del libro y la lectura es una de las áreas de trabajo tradicionales de las bibliotecas infantiles y debe seguir siéndolo, pero en la sociedad digital, profesionales y usuarios deben asumir que se puede leer en distintos formatos y de diferentes maneras, con o sin pantalla, y que se pueden hacer las mismas actividades utilizando también ambos formatos de forma independiente o integrada.

La tecnología ofrece nuevas posibilidades de dinamización que se pueden aprovechar para conseguir que los usuarios conozcan y disfruten con los nuevos contenidos, sin olvidar los tradicionales. 


\subsubsection{Formación E INSTRUCCIÓN}

El primer paso para dinamizar los contenidos digitales en bibliotecas pasa por la formación, ya que según la Asociación Internacional de Asociaciones Profesionales de Bibliotecas y Bibliotecarios (IFLA), en su informe “¿Surcando las olas o atrapados en la marea?” (IFLA, 2013), la formación y la adquisición de competencias digitales es esencial para la inclusión de los ciudadanos en el mundo digital.

La lectura digital implica nuevas habilidades y destrezas que son las que permitirán aprovechar al máximo las posibilidades de estos soportes: navegar por las páginas del libro, por el texto, la ilustración, los hipervínculos, los menús, etc. Es un tipo de lectura que supone un esfuerzo añadido para relacionar los componentes que conforman cada obra y, para ello, niños, jóvenes y adultos deben ser instruidos (GarcíaRodríguez, 2013). En este contexto existe una necesidad de intervención de la biblioteca en la formación tanto en el uso de tecnologías de la información, como de los dispositivos y las aplicaciones de lectura. El trinomio formación-dinamización-servicios se articula como el eje en torno al cual pivota la acción de la biblioteca para un uso óptimo de sus colecciones y un aprovechamiento gratificante y comprometido por parte de sus usuarios (Cordón-García, Alonso-Arévalo, Gómez-Díaz \& Linder, 2013)

Por todo ello, los bibliotecarios en esta era digital deben proporcionar no solo el acceso, sino también la orientación y formación en el uso de los recursos electrónicos a través de una amplia variedad de medios:

- Elaboración de tutoriales de aprendizaje, preferiblemente en formato vídeo, que estén siempre disponibles en la web de la biblioteca. Los tutoriales deben recoger todos los aspectos relacionados con la lectura, descarga y préstamo de los contenidos en sus diferentes modalidades y para cada uno de los posibles dispositivos de lectura: ordenador, ereader, tabletas, etc.

- Cursos y talleres de formación, presenciales o en línea, adaptados a los distintos tipos de usuarios. Aunque hablemos de bibliotecas infantiles el público de estas actividades no tienen que ser exclusivamente los menores, sino que también desde la biblioteca se puede ofrecer formación a padres, ya que los contenidos digitales para niños pueden entrañar cuestiones complejas (accesibilidad a los contenidos, control parental, configuración de dispositivos...) en las que muchas veces los padres también necesitan orientación. Y es que saber manejar un dispositivo no es lo mismo que saber cómo usarlo, que es lo que deben pretender enseñar estos cursos y talleres.

- Incorporación de un espacio de preguntas frecuentes (FAQ) en la web de la biblioteca. Estas preguntas y sus correspondientes respuestas deben ir dirigidas tanto a mediadores (padres, profesores y bibliotecarios) como a los niños, por lo que deben estar redactadas con un lenguaje cercano y sin abusar de tecnicismos innecesarios.

- Instrucción personalizada a los lectores sobre el manejo y funcionamiento de dispositivos, bien de forma presencial o en línea a través de la web de la biblioteca.

Todas estas actividades de formación no solo son acciones de dinamización, sino que también permitirán a la biblioteca dar a conocer sus contenidos en profundidad, que los usuarios sepan manejarlos mejor y aumentar su visibilidad.

\subsubsection{RECOMENDACIONES Y GUÍAS}

La recomendación de contenidos siempre ha sido una de las actividades tradicionales de la biblioteca infantil, la diferencia es que actualmente la variedad es mayor, como también son más las herramientas, sobre todo tecnológicas, que se pueden utilizar para ello (Gómez-Díaz y García-Rodríguez, 2015). Al igual que en los casos anteriores con la recomendación en sí, lo que pretendemos conseguir es la integración total, por lo que 
debe hacerse tanto en papel como en digital e incorporar todo tipo de contenidos. Estas acciones permitirán no solo dinamizar las colecciones, sino también darles visibilidad e integrarlas:

- Elaboración de booktuber realizados por los propios bibliotecarios o invitando a los lectores a hacer las recomendaciones, eligiendo, siempre que sea posible, títulos disponibles en los dos formatos, impreso y digital. Los booktubers forman parte de los denominados por LLuch (2015) epitextos virtuales públicos, y se han convertido en una herramienta esencial para la promoción del libro que la biblioteca puede aprovechar. La biblioteca, además de prestar los medios para elaborarlos (cámara), puede ofrecer el alojamiento (canal de vídeo, ya sea en Youtube o Vímeo) y ofrecer sus plataformas (web y redes sociales) para difundirlos. De esta forma podemos incluso crear nuestra propia biblioteca de booktubers.

- Fomentar la interacción de los usuarios a través de los catálogos 2.0. De este modo, junto con la información bibliográfica, se ofrecen recomendaciones y valoraciones de las obras realizadas por los propios lectores.

- Elaborar guías de lectura en las que se integren todo tipo de contenidos, ofreciendo por ejemplo títulos en digital y sus versiones en papel, o unas obras de cada tipo o libros app basados en obras ya existentes en papel, como por ejemplo la realizada por AppTk: Apps más allá de los límites del papel 1,2

Estas guías deben estar segmentadas por grupos y categorías de usuarios, incluidos también padres, bibliotecarios y profesores, los cuales podrán estar informados de esta forma de los mejores contenidos y posteriormente recomendarlos. Estas guías pueden presentarse en forma de revista electrónica en formato flip page, disponible en la plataforma Issuu (https://issuu.com/).

- Ubicar en sitios estratégicos contenidos que animen a la lectura; poner un fragmento con un código QR que apunte a un desarrollo mayor, una ilustración extraída de un libro que lleve a una recomendación. Estas son distintas ideas que consiguen dar visibilidad al tiempo que dinamizan la colección.

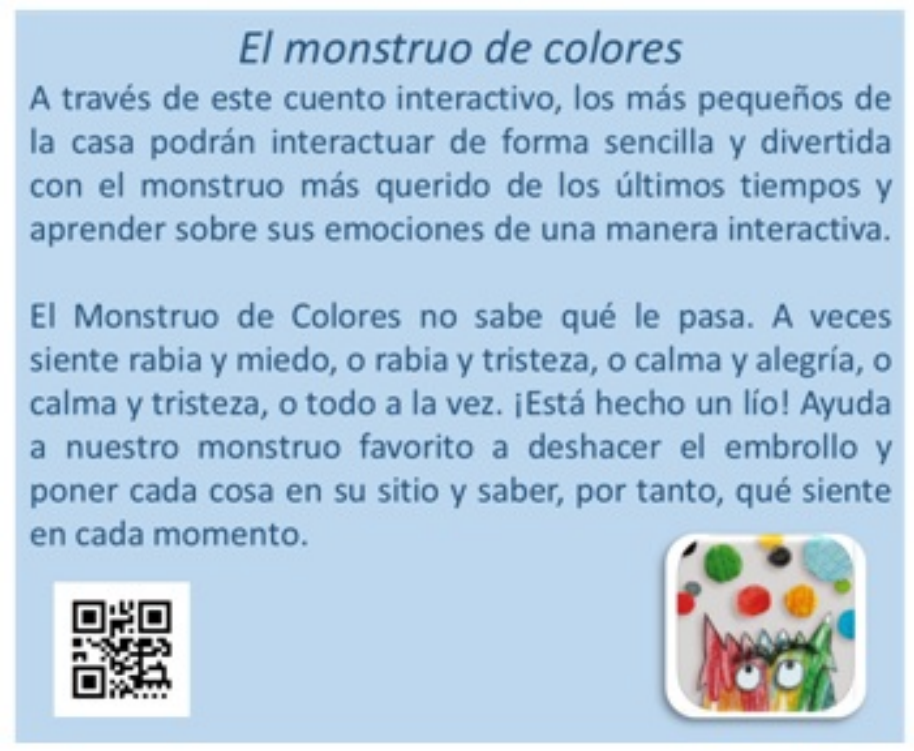

FIGURA 5

Cartel de recomendación con código QR

Fuente: Elaboración propia 
- Incorporar una sección específica en la web de la biblioteca siguiendo el modelo de Bibapps (http:// bibapps.com/), un recomendador de aplicaciones desarrollado por la Petite Bibliothèque Ronde. Incluso se podría diseñar una aplicación móvil específica que, a través de un sistema de alertas, informe al lector de la presencia de una nueva app en la biblioteca o una nueva recomendación en la web.

- Presentación de apps de lectura u otros contenidos digitales en la biblioteca, bien de forma esporádica o fijando un día concreto, por ejemplo al concluir las visitas escolares. Esta presentación se puede incluir también en las redes sociales de la biblioteca como "El libro digital del día" o "La app del día", etc.

- Realizar recomendaciones diarias de títulos, o con motivo de alguna celebración, centenario, etc. Estas recomendaciones se pueden hacer a través tarjetas con códigos QR creando "corners" en el espacio de la biblioteca, o bien generar centros de interés de los contenidos digitales.

En cualquiera de estas opciones, que por supuesto son complementarias, se deben seguir una serie de pautas: buscar un título llamativo pero lo suficientemente descriptivo (Apps que no te puedes perder antes de crecer); definir y sementar los posibles usuarios para orientar las recomendaciones a sus intereses; organizar y clasificar las recomendaciones, destacar los aspectos que más valora cada tipo de usuario (educativos en los adultos y lúdicos en los niños) e incluir una información lo más completa posible (Gómez-Díaz y GarcíaRodríguez, 2015).

\subsubsection{HORA DEL CUENTO}

Una de las formas más sencillas de dinamizar es la tradicional hora del cuento, en este caso digital, a la que podríamos denominar "APPS con mucho cuento", "App de cuento", "Había una vez una APP”, "CuentAPP”...

La interactividad y la posibilidad de personalización de las aplicaciones de lectura y libros app permiten amplias modalidades para la narración, como por ejemplo:

\section{Modalidad 1. Sesiones presenciales con libros digitales}

- Una opción es que el bibliotecario cuente la historia en voz alta y los niños vayan visualizando las imágenes en la pantalla. Para ello se pueden utilizar libros app (desactivando la locución y el texto); que el bibliotecario desarrolle su propio cuento a través de herramientas como Strorybook Reading u otras storytelling (CreAPPcuentos, Cada quien su cuento, Book Creator) o utilizar cuentos sin locución ni texto, como Marina y la Luz o los Tres Cerditos de Nosy Crow.

- La segunda opción consiste en utilizar la voz del narrador incorporada en la app al mismo tiempo que el texto se va reproduciendo en pantalla, o bien activar la locución, pero sin que aparezca el texto. Se podrían usar los cuentos incluidos en aplicaciones como Joy Tales, Mis cuentitos, Play Tales, o libros apps como Con le orecchie di lupo, Monster's Socks, Caperucita Verde, Lucy y Pogo o el Señor Lobo y las tortitas de jengibre.

\section{Modalidad 2. Sesiones presenciales con libros en papel y en digital}

Esta modalidad tiene como objetivo que los niños comprueben que se puede contar y disfrutar de la misma historia en los dos formatos. Podemos utilizar:

- Libros ya existentes en papel que se han desarrollado en forma de aplicación, o títulos protagonizados por personajes creados inicialmente para el papel como Olivia de Ian Falconer, Elmer de David Mckee, La pequeña oruga glotona de Eric Carle, Yo mataré monstruos por ti de Santi Balmes, El monstruo de Colores de Ana Llenas, Good Night, Little Rainbow Fish de Marcus Pfister, Hugless Douglas de David Melling o El Reino Subterráneo de Edward Packard. 
- Libros en papel con realidad aumentada: Los fantásticos libros voladores de Mr. Morris Lessmore, Les voyages fantastiques de Téo et Léonie, La Liebre y la Tortuga del desarrollador Arloon Kids, Un lobo boquiabierto o Hansel y Gretel de BooksARalive.

Las posibilidades de las apps son tantas que podemos incluso realizar sesiones de cuentacuentos para niños sordos, utilizando videolibros digitales o apps como TeCuento cuyainterfaz permite la visualización de vídeos en lengua de signos, de modo que una vez que hemos seleccionado un libro, texto e ilustración se complementan con el video signado. Igualmente se pueden utilizar audiolibros para niños ciegos o con dificultades de visión.

Durante el desarrollo de la sesión o al terminar la lectura, dependiendo de la aplicación que se utilice, se pueden realizar diferentes acciones que permitirán al niño participar de forma activa e interactuar no solo con la aplicación y su historia, sino también con el propio bibliotecario.

- Activación del movimiento de objetos y personajes, sonido, banda sonora, etc.

- Grabación personalizada de la historia por parte de los niños.

- Impresión de los personajes utilizando impresoras en $3 \mathrm{D}^{3}$.

- Cuentos del mundo: lectura de la historia en otras lenguas, aprovechando la posibilidad de cambio de idioma en la misma app.

Se recomienda seleccionar muy bien la aplicación, eligiendo aquellas que incorporen una buena historia, por supuesto, pero también que dispongan de buenas ilustraciones, bandas sonoras, locuciones de calidad y una interacción adecuada al texto y en un porcentaje proporcionado, utilizando para ello las fuentes y recursos determinados en la fase de selección de contenidos.

La tradicional hora del cuento se puede completar con talleres de escritura y/o ilustración de libros digitales. Una opción puede ser la trasposición de un libro en papel al formato digital utilizando aplicaciones como Book Creator, o crear cuentos originales basados en personajes existentes en libros impresos, generando contenidos con herramientas digitales.

\subsubsection{Club De LeCTURA}

Los clubs de lectura constituyen un tipo de práctica cultural y recreativa bastante usual en la actualidad. Desarrollan actividades que involucran a un número amplio de personas, que se reúnen frecuente y periódicamente a lo largo de periodos de tiempo muy extensos, y gozan de reconocimiento y prestigio entre la ciudadanía (Agustín Lacruz y Morera Gracia, 2016). Entre sus objetivos está el conseguir y fidelizar lectores en entornos agradables e integradores, en los que se propicie el crecimiento personal a través del aprendizaje continuo y el enriquecimiento del tiempo de ocio (Moreno Mulas, García-Rodríguez, y Gómez-Díaz, 2017).

En los clubs el entorno en el que se produce la conversación puede ser el físico, pero también virtual, y la materia que provoca dicha conversación puede ser digital o estar en papel; por tanto, son un lugar perfecto que favorece la integración de la colección, al tiempo que sirve para la dinamización.

Combinar los clubs de lectura presenciales utilizando un libro digital, desarrollarlo en la nube con obras impresas, o que cada lector elija el formato, puede ser una forma de dinamizar y visibilizar contenidos. Un ejemplo de club de lectura peculiar lo podemos encontrar en Librarium, una plataforma desarrollada por la Consejería de Educación y Empleo de la Junta de Extremadura, que se compone por un lado de una plataforma de préstamo y por otra de un club de lectura digital. Este proyecto tiene como objetivo potenciar el entorno virtual de las bibliotecas escolares para que colecciones físicas y digitales se complementen (https:// librarium.educarex.es/opac/\#indice). 
Experiencias de clubs de lectura con diferentes soportes se desarrollan también en la Casa del Lector, con programas como AppTreve3 (http://casalector.fundaciongsr.org/apptreve3/) o iY Q+? Club de Lectura Creativa en distintos soportes (http://casalector.fundaciongsr.org/y-q-club-lectura-creativa/).

\subsubsection{GAMIFICACIÓN}

En los últimos años se oye hablar mucho de la gamificación: si bien aplicada al mundo de la educación resulta novedosa, las bibliotecas infantiles llevan tiempo utilizando los juegos como estrategia de dinamización lectora. Muchos libros app incluyen juegos como complemento o como un elemento que permite desbloquear determinadas partes de la historia, como es el caso del libro app Swaptales: ¡León!, aunque lo más frecuente es la realización de juegos con personajes de los libros, a través de aplicaciones basadas en ellos o juegos que están incorporados en la misma app. El juego de Rita la Lagartija; La Pequeña Oruga Glotona, Gruffalo Games; El gran juego de Geronimo Stilton o Roald Dabl's Twit or Miss, pueden ser ejemplos muy interesantes.

Además de los juegos que incorporan los libros app o los juegos basados en libros, desde la biblioteca se pueden crear retos de lectura, a caballo entre el desafío y el juego, algo similar al desafío realizado desde Argentina por la plataforma Leer 20-20 (https://desafio.leer.org/).

En esta categoría se pueden incluir también campañas de difusión en forma de juegos y concursos al estilo de "Mi libro favorito", en la que los lectores están invitados a enviar fotografías suyas junto a su libro preferido en papel o en una tableta o smartphone (http://conectatealalectura.cl/).

\subsubsection{Préstamo}

El tradicional servicio de préstamo, de contenidos digitales o impresos, es una herramienta de dinamización que no se debe olvidar. Algunas posibles acciones son:

- Ofrecer recompensas cuando se preste en digital un libro que también está en papel. Puede diseñarse un álbum de cromos con pegatinas de las cubiertas de los libros que se darán al lector cada vez que elija un libro electrónico.

- Préstamo de tabletas cargadas con apps de lectura o libros, entre los cuales aparezcan también aquellos que estén en papel. De este modo, el lector podrá ver las posibles diferencias entre uno y otro formato y elegir el que más le interesa. Este tipo de experiencias se están desarrollando ya en algunos centros como La Casa del Lector de la Fundación Germán Sánchez Ruipérez, o las Maletas Digitales de la Biblioteca Artur Martorell. Estas tabletas pueden estar disponibles en las máquinas de autopréstamo como se realiza en la biblioteca universitaria de Drexel (Drexel University Library) o realizar el préstamo domiciliario de dispositivos como el programa Homebound Services de la Rapid City Public Library en Dakota del sur (https://www.freelibrary.org/). 


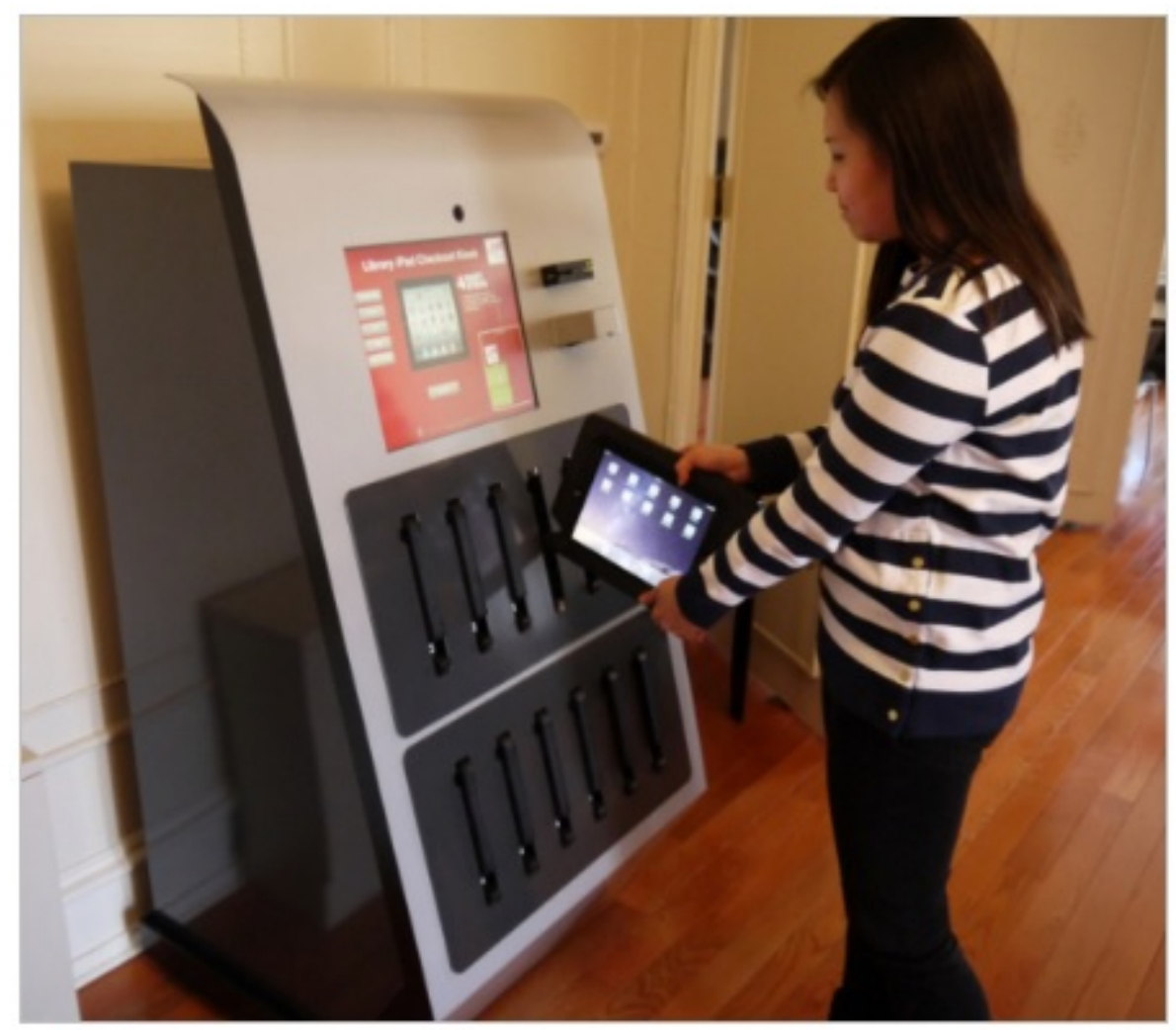

FIGURA 6

Préstamo de dispositivos en bibliotecas

Fuente: http://appleinsider.com/articles/15/04/02/drexel-universityinstalls-ipad-rental-vending-machine-for-students-library-card-holders

- Préstamo de packs de libros impresos y digitales en los que, junto a una selección de libros impresos, se preste una tableta con esos mismos títulos en formato app.

- Préstamo digital desde las instalaciones físicas de la biblioteca o en cualquier punto de la ciudad, estaciones de metro, marquesinas de autobuses, parques... Pueden ser un lugar para colocar beacons y permitir el préstamo a través del móvil.

\section{A MODO DE CONCLUSIÓN}

Las bibliotecas deben mirar al futuro viviendo el presente y teniendo muy claro cuál es su pasado. Nos enfrentamos a nuevos usuarios, nuevos formatos, soportes, formas de comunicación, y a retos y oportunidades que no debemos dejar escapar. No todo en la biblioteca va a pasar por la tecnología, pero gran parte de nuestro trabajo se va a centrar en ella y no podemos mirar para otro lado.

Seleccionar los mejores contenidos digitales, saber recomendarlos, difundirlos y utilizarlos en las actividades habituales de la biblioteca, es uno de los retos que los bibliotecarios infantiles del siglo XXI deben asumir. Ello no significa que tengan que desaparecer las actividades tradicionales. La oferta dirigida a los niños y jóvenes se puede enriquecer con actividades que incorporen dispositivos y contenidos digitales, que se desarrollen en el espacio físico de la biblioteca y actividades en las que los contenidos en papel tengan cabida en el ámbito digital.

Tal como afirma Cencerrado-Malmierca (2017), es preciso dar un paso adelante y romper la dicotomía entre el papel y la pantalla en favor de un concepto amplio y completo de lectura que abarque todas las aristas 
que la lectura presenta en la actualidad. De esta manera podremos abordar con mayor fortaleza y alcance los retos que plantean los lectores, promover y apoyar nuevos hábitos de lectura.

Necesitamos lectores que conozcan y se muevan entre todo tipo de textos y formatos y que estén preparados para ir de uno a otro de manera natural.

La labor dinamizadora de la biblioteca en la sociedad actual consiste en aunar lo analógico y lo digital de modo que ambos formatos se complementen y se integren de forma única, desarrollando estrategias de promoción conjunta y así fomentar la visita a la biblioteca física partiendo del mundo digital.

Viejas y nuevas experiencias de lectura, en definitiva, que se complementan, que enriquecen las experiencias lectoras, que se deben formular como suma y no como resta, que multiplican las posibilidades de acceso a la cultura (Cencerrado-Malmierca, 2017).

\section{REFERENCIAS}

Agustín Lacruz, M. del C. A. y Morera Gracia, E. (2016). Los clubes de lectura en Aragón: análisis descriptivo de una práctica socio-cultural de animación y promoción lectora. Revista General de Información y Documentación. Gen. Inf. Doc, 26(2), 583-603. https://doi.org/10.5209/RGID.54716

Alonso-Arévalo, J., Gómez-Díaz, R. y Cordón-García, J. A. (2015). Ebooks en bibliotecas: gestión, tratamiento y aplicaciones. Buenos Aires: Alfagrama.

Aprovechar las TIC en la biblioteca infantil. (2016). Recuperado de http://www.lecturalab.org/story.php?id=2792

Cencerrado-Malmierca, L. M. (2017). En el cruce de los viejos y los nuevos caminos que invitan a leer. Anuario Think EPI, 11. https://doi.org/10.3145/thinkepi.2017.31

Comalat-Navarra, M. (2015). Bibliotecas públicas e impresoras 3D: el debate está servido. El Profesional de la Información. https://doi.org/10.3145/epi.2015.mar.12

Cordón-García, J. A. (2016). La lectura digital y la formación del lector digital en España: la actividad de la Fundación Germán Sánchez Ruipérez y el Proyecto Territorio Ebook. Alabe Revista de Investigación sobre Lectura y Escritura, 7(13), 1-25. https://doi.org/10.15645/Alabe2016.13.11

Cordón-García, Alonso-Arévalo, J., Gómez-Díaz, R. y Linder, D. (2013). Social Reading: Platforms, Applications, Clouds and Tags. Social Reading: Platforms, Applications, Clouds and Tags. https://doi.org/10.1108/OIR-08-2 014-0186

E-book collection management in UK university libraries: focus groups report by Jisc Collections - issuu. (2009). Recuperado de http://issuu.com/carenmilloy/docs/libraryfocusgroups?mode=embed\&viewMode=presentati on\&layout=http\%3A\%2F\%2Fskin.issuu.com\%2Fv\%2Flight\%2Flayout.xml\&showFlipBtn=true

El carrusel de portadas de títulos llega a los catálogos de las bibliotecas. (2017). Recuperado de http://www.comunidadbaratz.com/blog/el-carrusel-de-portadas-de-titulos-llega-a-los-catalogos-de-las-bibli otecas/?utm_source=acortador\&utm_medium $=$ hootsuite\&utm_campaign=acortadorhootsuite

Gallo León, J. P. (coord.). (2013). Prospectiva 2020: las diez áreas que más van a cambiar en nuestras bibliotecas en los próximos años. Biblioteques de Barcelona, 1-92. https://doi.org/http://hdl.handle.net/10421/7461

García-Rodríguez, A. (2013). Visibilidad de la literatura infantil y juvenil en las plataformas de distribución y venta de libros electrónicos. En J. A. Cordón-García, R. Gómez-Díaz y J. Alonso-Arévalo (Eds.), Documentos electrónicos y textualidades digitales: nuevos lectores, nuevas lecturas, nuevos géneros (pp. 145-158). Salamanca: Universidad de Salamanca.

García-Rodríguez, A. y Gómez-Díaz, R. (2016a). Contenidos enriquecidos para niños o las nuevas formas de leer, crear y escuchar historias: una propuesta de clasificación. Revista Chilena de Literatura, 94, 173-195. https://doi.or $\mathrm{g} / 10.4067 / \mathrm{S} 0718-22952016000300009$

García-Rodríguez, A. y Gómez-Díaz, R. (2016b). Lectura digital infantil: dispositivos, aplicaciones y contenidos. Barcelona: UOC y El profesional de la Información. 
García-Rodríguez, A. y Gómez-Díaz, R. (2017a). Literatura digital infantil y juvenil en tabletas y smartphones: una oportunidad para lograr nuevos lectores. Anuario ThinkEPI, 11 (0), 167. https://doi.org/10.3145/thinkepi.20 17.30

García-Rodríguez, A. y Gómez-Díaz, R. (2017b). Más allá del «había una vez». En R. Gómez-Díaz, A. GarcíaRodríguez y J. A. Cordón-García (Eds.), Fuentes especializadas en Ciencias Sociales y Humanidades. Madrid: Pirámide.

Gómez-Díaz, R. y García-Rodríguez, A. (2015). La biblioteca infantil en el siglo XXI o cómo integrar las apps en la biblioteca. Prólogos. Revista de Historia, Política Y Sociedad, VII(2014-2015), 103-131. Recuperado de https:/ /gredos.usal.es/jspui/bitstream/10366/130152/1/2015Prologos-VII-GDiazGRodriguez.pdf

Gómez-Díaz, R., García-Rodríguez, A., Cordón-García, J. A. y Alonso-Arevalo, J. (2016). Leyendo entre pantallas. Ediciones Trea.

IFLA. (2013). Surcando las olas o atrapados en la marea. Trends.Ifla.Org. Recuperado de https://trends.ifla.org/files /trends/assets/ifla-trend-report_spanish.pdf

Lluch, G., Tabernero-Sala, R., Calvo-Valios, V. (2015). Epitextos virtuales públicos como herramientas para la difusión del libro". El profesional de la información, 24(6).797-804. Recuperado de http://www.elprofesionaldelainform acion.com/contenidos/2015/nov/11.pdf

Marquina, J. (2013). Informe Apei sobre Bibliotecas ante el siglo XXI: nuevos medios y caminos, Apei, 8 Recuperado de https://www.julianmarquina.es/informe-apei-bibliotecas-ante-el-siglo-xxi-nuevos-medios-y-caminos/

Moreno Mulas, M. A., García-Rodríguez, A. y Gómez-Díaz, R. (2017). Conversando en la nube: cómo organizar un club de lectura virtual. Revista General de Información y Documentación, 27(1), 177-200. https://doi.org/10.5 209/RGID.56566

\section{Notas}

1 http://apptk.es/apps-mas-alla-de-los-limites-del-papel-y-la-pantalla/

2 Otros ejemplos de guías de este tipo son las de la Fundación Germán Sánchez Ruipérez (http://recursos.fgsr.es/lomas/ index.html), el Grupo de Investigación Gretel (http://www.literatura.gretel.cat/es), las recogidas en la sección recursos en Lectura Lab (http://lecturalab.org/section/RECURSOS-Y-CURSOS_27) o el blog de Consultoría de Cultura y Lectura de Elisa Yuste http://www.elisayuste.com/blog/.

3 Las impresoras 3D en la biblioteca ofrecen muchas posibilidades, como por ejemplo la impresión de cuentos en 3D para niños con dificultades visuales, como se está haciendo en http://www.tactilepicturebooks.org/. 